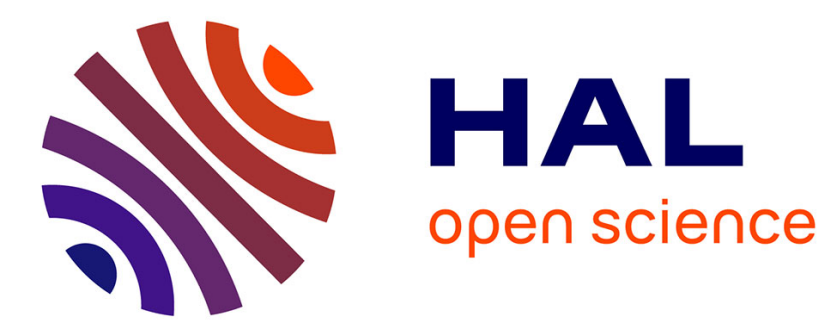

\title{
Throughput-Maximizing Transmission Schedules for Underwater Acoustic Multihop Grid Networks
}

\author{
Said Lmai, Mandar Chitre, Christophe Laot, Sébastien Houcke
}

\section{To cite this version:}

Said Lmai, Mandar Chitre, Christophe Laot, Sébastien Houcke. Throughput-Maximizing Transmission Schedules for Underwater Acoustic Multihop Grid Networks. IEEE Journal of Oceanic Engineering, 2015, 40 (4), pp.853 - 863. 10.1109/JOE.2015.2474455 . hal-01224554

\section{HAL Id: hal-01224554 \\ https://hal.science/hal-01224554}

Submitted on 11 Jun 2021

HAL is a multi-disciplinary open access archive for the deposit and dissemination of scientific research documents, whether they are published or not. The documents may come from teaching and research institutions in France or abroad, or from public or private research centers.
L'archive ouverte pluridisciplinaire HAL, est destinée au dépôt et à la diffusion de documents scientifiques de niveau recherche, publiés ou non, émanant des établissements d'enseignement et de recherche français ou étrangers, des laboratoires publics ou privés. 


\title{
Throughput-maximizing Transmission Schedules for Underwater Acoustic Multihop Grid Networks
}

Said Lmai, Member, IEEE, Mandar Chitre, Senior Member, IEEE, Christophe Laot, Senior Member, IEEE, and Sebastien Houcke, Member, IEEE,

\begin{abstract}
Many marine scientific, industrial and military applications may require the deployment of underwater acoustic sensor networks for sensing and monitoring. A grid topology with multihop relaying is useful for wide area coverage as well as long distance data transmission. We investigate network architectures where data originate at one end of the grid, and are forwarded along multiple lines. We are particularly interested in transmission schedules that maximize network throughput by exploiting propagation delay to allow multiple simultaneous transmissions. We show that an optimal schedule is necessarily per-node fair, and derive the upper bound on throughput. Furthermore, we present a low-complexity algorithm to find schedules achieving the upper bound, regardless of the size of the network.
\end{abstract}

\section{INTRODUCTION}

Applications of underwater acoustic (UWA) sensor networks include scientific exploration (e.g., to observe marine biology or ocean floor activity), industrial monitoring (e.g., to monitor and manage commercial fishing activities or undersea oil extraction), and military missions (e.g., to secure sensitive areas like port facilities or to monitor ships in foreign harbors) [1]. When the area involved is large, multiline grid topologies with multihop relaying may be considered, particularly for high-rate and long distance communication services. Multiline grid topology consists of several parallel lines of regularly placed nodes. On each line, messages originating from the first node are relayed hop-by-hop until they reach the final destination node at the extremity of the line. Indeed, in applications related to UWA sensor networks, we have two major parameters to consider: (1) the transmission range, which depends on bandwidth and power [2]; and (2) the extent of coverage, which depends on the need and budget. A potential deployment could consist of a set of nodes (e.g., seismic sensors) installed along a tsunami risk zone [3] that would monitor the movement of the wave, i.e., the tsunami path, over a large area. In addition to environmental monitoring applications, another practical use of the proposed grid topology would be to secure areas where military exercises and operations are held, or where critical ocean infrastructure is deployed. We focus on applications where a regular grid topology is used. Without loss of generality, we depict the direction of relaying vertically in our illustrations, and assume the spacing between neighboring nodes on the same line to be one unit. We specifically focus on a 
multiline grid topology (see Fig. 1) where the distance separating every two adjacent lines is two units. The essential features of the considered grid topology are provided in detail in section IV-B.

As in all shared-medium networks, a medium access control (MAC) protocol is necessary to regulate and coordinate UWA channel access. Since our sensor networks are assumed to generate data at a regular rate, we consider a scheduled MAC rather than a random-access MAC. Scheduled MAC protocols do not waste energy on collisions and handshaking, and hence are more energy-efficient than random-access MAC protocols [4]. As propagation delays in UWA networks are large, the traditional scheduled time division multiple access (TDMA) protocol suffers from low performance due to the long guard time required. We use a variant of TDMA where packet transmissions can overlap (thus reducing or eliminating guard times) without colliding at a receiver. This allows us to use propagation delay constructively, to maximize network throughput.

In this paper, we show how large propagation delays in UWA multihop grid networks may be exploited, in an unconventional approach, to achieve high channel utilization. Specifically, in a grid topology as outlined above, we are interested in TDMA-based transmission schedules that take advantage of long propagation delays in order to optimize the overall network throughput. To the best of our knowledge, such a study has not been undertaken previously in the context of multihop grid topologies. We prove that an optimal periodic schedule in a regular multiline grid network with multihop relaying is necessarily per-node fair. Furthermore, we derive the upper bound on network throughput. We then propose schedules to achieve the upper bound and present a computationally efficient algorithm for developing such schedules. The schedules are designed to allow as many simultaneous transmissions as possible, while limiting interference at unintended nodes.

The importance of grid topologies in an underwater environment has been recognized by several researchers, and such topologies have been the subject of inquiry in many studies. The authors in [5] investigate the physical characterization of a multihop cooperative communication in a grid network topology. In [6], the authors present a networking protocol for node discovery and localization. A grid structure is considered in [7] where the authors investigate the design of an optical underwater sensor network. Three separate grid arrangements were tested in [8], using radio frequency electromagnetic communication in a small scale underwater wireless sensor network. However, to the best of our knowledge, no effort has been made previously towards the development of TDMA-based MAC schedules.

Most linear multihop network topology studies have focused on the physical link. Analysis in [2] takes into account interhop interference and shows achievable information rates versus per-node power. Nevertheless, no network-oriented performance is explored. In [3], multihop linear topology is explored under fair access criterion for all nodes. Identical distance separates every two neighboring nodes. The transmission range for each node is assumed to be one hop, while the interference range is less than two hops. In the case where the message duration is set to be same as the one-hop propagation delay (as we do in this paper), the authors in [3] derive an upper bound in terms of overall network utilization (defined as the fraction of time that the final destination node is busy receiving messages). The linear topology can be seen as a particular case of multiline grid topology. Although we assume the interference range to be twice the transmission range, we derive optimal schedules achieving a tighter 
upper bound for network utilization.

In the literature, TDMA-based MAC protocols in underwater networks is still an area that has not be extensively investigated using analytical models for the optimization process. In [9], the authors introduce an optimal traffic scheduling solution using a weighted, directed conflict graph with the frame size minimizing and network throughput maximizing target in a fully-connected network. Using a color (an integer) assigned to each edge in the conflict graph, transmissions are scheduled. In view of the complexity of the corresponding problem, an approximate algorithm based on a greedy heuristic of the vertex coloring problem is proposed. Yet, in a fully-connected network with one final destination, the authors of [10] reduce the scheduling problem to a standard traveling salesman problem. However, the optimal solution found for is still complex to implement and does not describe the steady state for routing and scheduling. In a multihop scenario where a sink node is collecting all the information coming from the sensors, the authors in [11] propose an energy consumption minimization model, which addresses routing and scheduling in small underwater networks, with a degree of liberty as regards nodes' placement. In a similar way, the authors in [12] present an analytical model for joint MAC and routing optimization in small to medium scale networks. The authors impose a periodic scheduling of transmissions from the nodes. Both works use heuristics and consider the presence of multiple interfering nodes and the use of an underwater acoustic channel attenuation model. The results obtained in [12] using heuristic prediction are close to the optimum derived using the adopted model. In this paper, we have multiple destination nodes in a multihop network with a grid topology. Even if, we do not consider physical link features, we analytically derive, at a MAC sub-layer level, the upper bound on the network throughput. Furthermore, we use heuristics in a practical and very low-complexity algorithm that exactly lead to the optimum performance. Moreover, with our solution, we do not need to enforce the periodicity constraint in order to reach the steady state of scheduling.

The idea of taking advantage of large propagation delays is not new (e.g. [13]-[16]). However, the demonstrated performance in terms of normalized network throughput does not exceed 1 regardless of the network topology adopted. Yet the authors in [17] show that within one collision domain, an $N$-node network may achieve a throughput of up to $N / 2$. In multihop networks, interference is limited as compared to a single collision domain network, and therefore we may expect an ever larger throughput. We use the valuable results from [17] to conduct a study with sharper focus - a multiline grid topology. Our work shows that a larger throughput is indeed achievable through careful scheduling of transmissions from each node in the network.

The remainder of the paper is organized as follows. Section II describes the general context and system model. In section III, we derive an upper bound on the network throughput. In section IV, we propose schedules achieving the upper bound and present in section $\mathrm{V}$ a computationally efficient algorithm to find optimal schedules. Section VI concludes the paper.

\section{SySTEM MODEL}

In a nonzero propagation delay environment, we consider a regular $N$-node network with multiline grid topology where each node is identified by $i$ s.t. $i \in \mathbb{N}^{*}$ and $1 \leq i \leq N$. Let $\eta \geq 1$ denote the number of independent node 


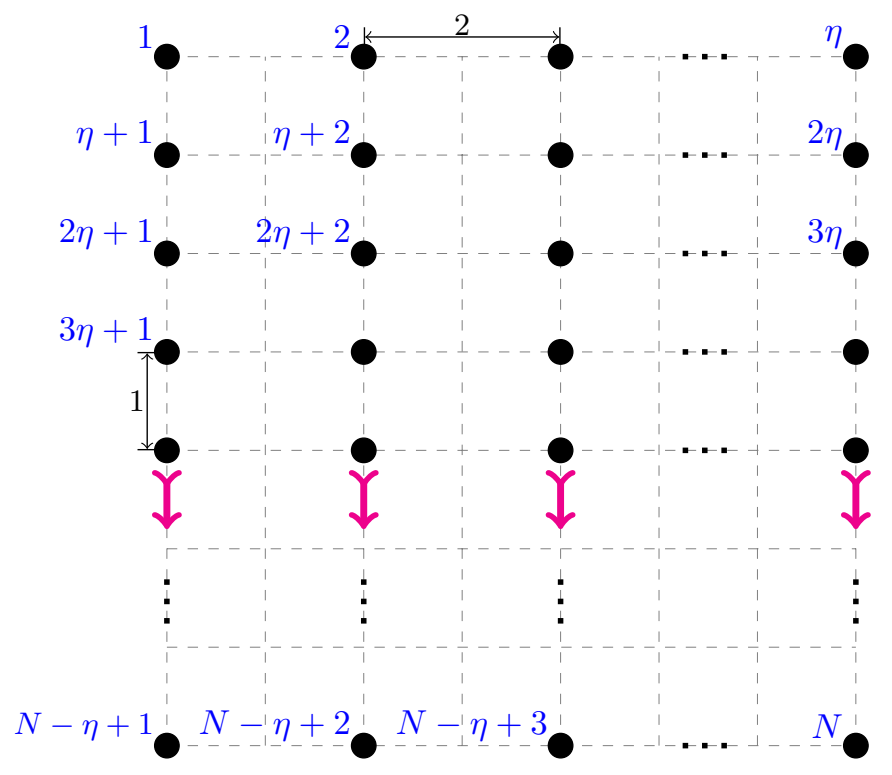

Fig. 1. A regular $N$-node multiline grid network.

lines in the network. Messages originating from nodes $1,2, \cdots, \eta$ are relayed hop-by-hop until they reach final destination nodes $N-\eta+1, N-\eta+2, \cdots, N$, respectively. An illustration of this architecture is shown in Fig. 1. Note that, in all figures, node lines are graphically represented by the columns.

Let $\mathbf{r}_{i}$ be the position vector of node $i$ in 3-D Euclidean space. The propagation delays between every pair of nodes may be expressed using a delay matrix (see [17]) denoted by D. Time is assumed to be slotted. Thus,

$$
D_{i j}=\frac{\left|\mathbf{r}_{i}-\mathbf{r}_{j}\right|}{c \tau}, \quad 1 \leq i, j \leq N
$$

where $c$ is the signal propagation speed and $\tau$ is the length of one time slot. The entries of $\mathbf{D}$ are nonnegative real numbers. Thus, between every pair of nodes $(i, j)$, where $i \neq j$, there is a nonzero propagation delay $D_{i j}$. The geometry of the network is fully characterized by the delay matrix $\mathbf{D}$. Moreover, $\mathbf{D}$ is symmetric i.e., $D_{i j}=D_{j i}$, and it has an all-zero diagonal i.e., $D_{i i}=0$.

In this paper, our focus is on a network with unit spacing between every pair of neighboring nodes on the same line. We set $\tau$ to the propagation delay between two neighboring nodes on the same line. The distance separating two adjacent node lines is equal to 2 units. Accordingly, in the grid structure of the network, vertical span is 1 unit while the horizontal span is 2 units.

Although weak signals cannot be successfully decoded, they may cause interference. In wireless radio networks, the interference range is often considered to be approximately twice the transmission range [18], [19]. We set the transmission range $g$ of each node in the network to 1 , and allow the interference range to be 2 . Beyond this range, the interference is considered to be too weak to cause packet loss. An interference range of 2 represents a worst-case scenario. Yet, with the grid structure of the network, a slightly higher interference range can be accommodated 


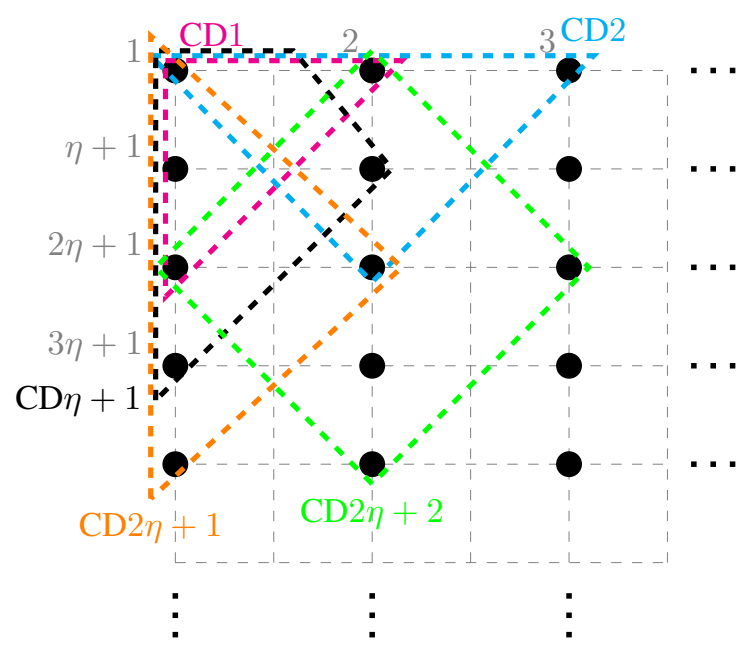

Fig. 2. Some partially-overlapping collision domains.

without any change to the analysis.

A collision domain is identified with respect to each node in the network. We then have $N$ partially-overlapping collision domains. With regards to packet delivery, unicast traffic is used, i.e., a message is sent from a single source node to a single destination node. Except for the source and destination nodes, a message is considered as an interference at all other nodes that it reaches. Fig. 2 shows some collision domains. CD $k$ is used to designate the collision domain relative to node $k$. All nodes are assumed to operate in half-duplex mode, i.e., a node cannot simultaneously transmit and receive.

Assuming the physical link to be reliable (error free) with constant data rate $\nu$, the loss of a message is due only to collision. A collision is said to occur at a certain node if two or more messages overlap in time. A successful transmission refers to a transmission that results in a successful reception of the message at the destination node. The normalized network throughput (henceforth simply called network throughput or throughput) $Y$ is the total number of information bits successfully received by all nodes in the network per unit time, normalized by the link data rate $\nu$.

Provided that the message duration is equal to $\tau$, we define a transmission schedule $\mathbf{S}$ as the matrix that determines when each node transmits and receives messages. We follow the same convention as [17], where the entries of $\mathbf{S}$ correspond to the different scenarios below:

- $S_{i t}=S_{i, t}=j>0$ indicates that node $i$ transmits a message to node $j$ at time slot $t$.

- $S_{j t}=S_{j, t}=-i<0$ implies that node $j$ receives a message from node $i$ during the time slot $t$.

- In all other cases, node $i$ is designated as an idle node during time slot $t$, which is represented by $S_{i t}=S_{i, t}=0$.

If $S_{i, t+T}=S_{i t} \forall i, t$, the schedule is repeating with a period $T$. Such periodic schedules are depicted using an 


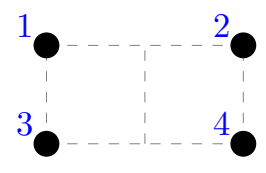

Fig. 3. A 2-line grid 4-node network.

$N \times T$ matrix $\mathbf{S}^{(T)}$ where

$$
S_{i t}=S_{i, t}^{(T)}(\bmod T)
$$

Node $i$ transmits a message to node $j$ during time slot $t$ only if node $j$ is able to successfully receive the message during time slot $t+D_{i j}$, i.e.,

$$
S_{i t}=j \quad \Leftrightarrow \quad S_{j, t+D_{i j}}=-i \quad \forall i \neq j, D_{i j} \leq g
$$

Furthermore, to ensure the successful reception at time slot $t$ of a transmitted message, it is required that no other nodes transmit messages that arrive at node $j$ during $t$. Thus,

$$
S_{j t}=-i \quad \Rightarrow \quad S_{k, t-D_{j k}} \leq 0 \quad \forall k \neq i, D_{j k} \leq 2 g
$$

From $\mathbf{S}^{(T)}$, we can calculate the average network throughput considering the number of receptions in $\mathbf{S}^{(T)}$ :

$$
Y=\frac{1}{T} \sum_{t=0}^{T-1} \sum_{i=1}^{N} \mathbb{1}\left(S_{i t}^{(T)}<0\right),
$$

where $\mathbb{1}(E)$ is the indicator function of the event $E$, with value of 1 if $E$ is true and 0 otherwise.

By way of illustration, consider the following schedule in the regular 2-line grid 4-node network (described in Fig. 3):

$$
\mathbf{S}^{(2)}=\left[\begin{array}{cc}
3 & 0 \\
4 & 0 \\
0 & -1 \\
0 & -2
\end{array}\right] .
$$

The delay matrix defining the geometry of this 4-node grid network is:

$$
\mathbf{D}=\left[\begin{array}{cccc}
0 & 2 & 1 & \sqrt{5} \\
2 & 0 & \sqrt{5} & 1 \\
1 & \sqrt{5} & 0 & 2 \\
\sqrt{5} & 1 & 2 & 0
\end{array}\right] .
$$

An explanation of the transmissions and the corresponding receptions handled in $\mathbf{S}^{(2)}$ is shown in Table I. Note that the network throughput in this case is $Y=1$. If the columns of the matrix $\mathbf{S}^{(2)}$ are circularly shifted to the right or to the left, the resulting matrix describes the same transmission schedule.

Note that, as in [20], since we only use delays on a fundamental level, our approach cannot exploit the capture effect, the effect of receiving correctly a packet from a collision, i.e., even in the presence of other concurrent 
TABLE I

SUMMARY OF PLANNED TRANSMISSIONS AND THE CORRESPONDING RECEPTIONS IN ACCORDANCE WITH $\mathbf{S}^{(2)}$

\begin{tabular}{|c|c|c|}
\hline \hline Node $\backslash$ time slot & time slot $\mathbf{1}$ & time slot $\mathbf{2}$ \\
\hline Node $\mathbf{1}$ & Transmission to node 3 & Idle \\
\hline Node $\mathbf{2}$ & Transmission to node 4 & Idle \\
\hline Node $\mathbf{3}$ & Idle & Reception from node 1 \\
\hline Node $\mathbf{4}$ & Idle & Reception from node 2 \\
\hline
\end{tabular}

transmissions. However, our study can be extended in the way to take advantage of the capture effect, as in [21], in a future work.

As introduced in [17], when a schedule $\mathbf{S}^{(T)}$ provides the same number of transmission opportunities to all nodes, $\mathbf{S}^{(T)}$ is said to be per-node fair. This can be written as:

$$
\sum_{t=0}^{T-1} \mathbb{1}\left(S_{i t}^{(T)}>0\right)=\text { constant }>0 \quad \forall i .
$$

Note that the final destination nodes are not included in this fairness characteristic, since they either receive or remain idle at any time.

\section{Achievable Throughrut}

We define an optimal transmission schedule as being the schedule that maximizes network throughput. We start by showing an important feature of such a schedule.

Theorem 1. An optimal periodic transmission schedule in a regular $N$-node multiline grid network with multihop relaying is necessarily per-node fair.

Proof: Since every network has an optimal schedule that is periodic [17, Theorem 6], we are interested in schedules $\mathbf{S}^{(T)}$ with period $T$.

Let us first distinguish different classes of interferences ${ }^{1}$ that one should deal with in the most restrictive scenario. We can regard two interferences (in the same direction) overlapping at a certain node as one interference, since their impact on that node is exactly the same. For example, in Fig. 4, two interferences at node $\eta$ have the same effect as one interference at node 1 . Note that since each transmission takes exactly one time slot, the message coming from the two-hop neighbor at time slot $t-1$, overlaps with the message coming from the one-hop neighbor at time slot $t$.

CASE 1 (1-interference): The final destination nodes do not transmit, and each of them can be affected by one interference coming from the 2-hop downstream node on the same line. In Fig. 4, nodes $N-\eta+1$ and $N$ are two illustrative examples of this case.

\footnotetext{
${ }^{1}$ For the sake of brevity and clarity, we use the word "interference" as a countable noun to mean "the arrival of an interfering transmission".
} 


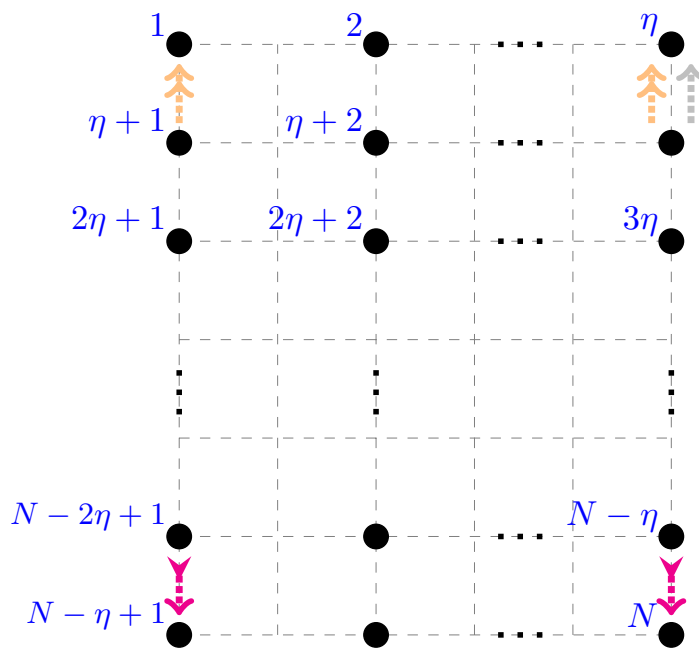

Fig. 4. The impact of 1-interference in the network.

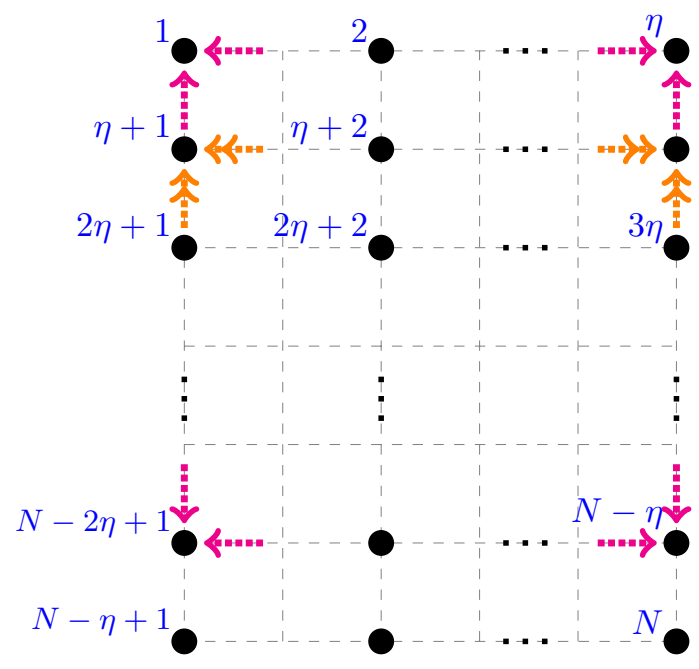

Fig. 5. The impact of 2-interferences in the network.

CASE 2 (2-interferences): This case involves the two first nodes and the last-but-one node on a line at the edge. In Fig. 5, an illustration of this situation is shown. The six nodes involved $(1, \eta, \eta+1,2 \eta, N-2 \eta+1$, and $N-\eta)$, can receive at most two interferences each from their neighbors during a time slot.

CASE 3 (3-interferences): This case involves the following nodes:

- all nodes in a line at the edge, except for the first two nodes and the last two nodes;

- the first two nodes and the last-but-one node on an inner line.

From the illustration shown in Fig. 6, we see that for the line at the left edge, the nodes between $2 \eta+1$ and $N-3 \eta+1$ (included) receive 3 interferences. On the line at the right edge, the nodes between $3 \eta$ and $N-2 \eta$ 


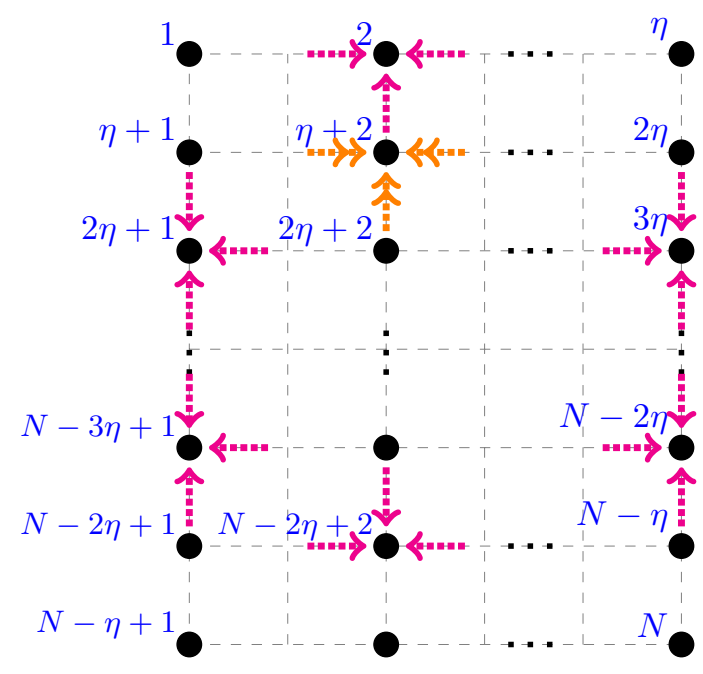

Fig. 6. The impact of 3-interferences in the network.

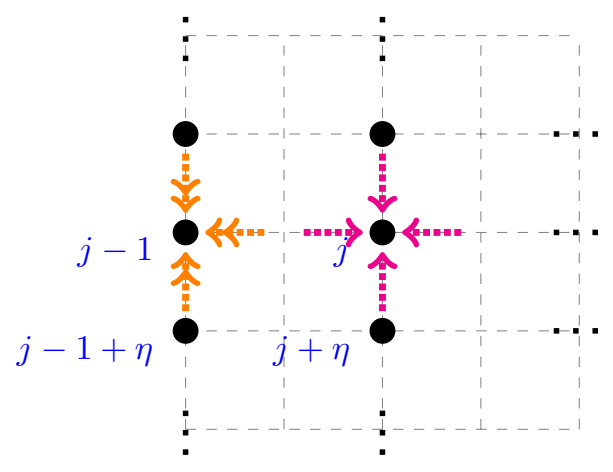

Fig. 7. The impact of 3- and 4-interferences in the network.

(included) receive 3 interferences. However, if we consider the first inner line from the left, the nodes that receive 3 interferences are $2, \eta+2$, and $N-2 \eta+2$.

CASE 4 (4-interferences): All nodes possibly impacted by four interferences belong to an inner line. Except for the first two nodes and the last two nodes, all nodes in an inner line are part of this case. Node $j$ in Fig. 7 serves as an illustrative example.

Let us assume that there is a transmission schedule $\mathbf{S}^{(T)}$ with at least two nodes $\bar{i}$ and $\bar{j}$ on the same line such that $\sum_{t=0}^{T-1} \mathbb{1}\left(S_{\bar{i} t}^{(T)}>0\right)>\sum_{t=0}^{T-1} \mathbb{1}\left(S_{\bar{j} t}^{(T)}>0\right)$, i.e., $\bar{i}$ transmits more often than $\bar{j}$. Let us consider nodes $i$ and $j$ where

$$
D_{i j}=\min _{\bar{i}, \bar{j}} D_{\bar{i} \bar{j}}
$$

Accordingly, $i$ and $j$ are neighboring nodes.

Consider the case where $i<j$. Since $i$ and $j$ are neighbors, $j=i+\eta$. A node $\bar{i}$ has, at most, three interfering 
neighbors if $\bar{i}$ belongs to an edge line, or four interfering neighbors if it belongs to an inner line. Examples are provided in Fig. 6 and 7 respectively. In order to find an optimal transmission schedule, one should find the appropriate strategy to manage interference. Nevertheless, we note that the presence of one interference at a certain node during a given time slot has exactly the same effect as the presence of two, three or four interferences - the node in question is not able to receive in that time slot as long as there is interference. Node $j$ is not the first node on the line since it receives from $i(<j)$. Then $j$ is impacted by interference from one, two, three or four surrounding nodes. Node $j+\eta$ is similarly impacted by interference from, at most, four surrounding neighbors. However, over one period $T, i$ transmits $n_{i T}=\sum_{t=0}^{T-1} \mathbb{1}\left(S_{i t}^{(T)}>0\right)$ messages, i.e., $j$ receives $n_{i T}$ messages, while $j+\eta$ receives only $n_{j T}<n_{i T}$ messages, where $n_{j T}=\sum_{t=0}^{T-1} \mathbb{1}\left(S_{j t}^{(T)}>0\right)$. In other words, even if nodes $j$ and $j+\eta$ are impacted in the same way by the interference, they do not receive the same amount of messages. This contradicts the objective of any strategy aiming to maximize the network throughput in such a regular structure.

Consider the case where $i>j$, i.e., $i=j+\eta$. Over one period $T$, node $i$ receives from node $j$ exactly $n_{j T}$ messages, while $i$ transmits $n_{i T}>n_{j T}$ messages. This indicates that $i$ transmits more messages than it has received from its downstream neighbor - an action which contradicts the multihop relaying concept adopted in the network.

Alternatively, nodes $\bar{i}$ and $\bar{j}$ could belong to two separate lines $L 1$ and $L 2$. If there exist nodes in $L 1$ or $L 2$ that do not transmit the same number of messages over one period $T$, the above demonstration still holds. Otherwise, we have two lines where all nodes transmit the same number of messages on each line. However, the total number of messages on $L 1$ and the total number of messages on $L 2$ may be different. Consider the most unfavorable case where $L 1$ is an edge line while $L 2$ is an inner line. The key factor in maximizing the throughput is planning the arrival of the maximum number of interferences at the time slot that will be used by the node for transmitting. In Fig. 7, one can observe that managing three interferences, like those affecting node $j-1$, or four interferences, like those affecting node $j$, may be performed in exactly the same way. Indeed, the node cannot receive in the presence of one or more interferences. Given the regular network geometry, any strategy adopted to maximize throughput on a single line should act identically on all other lines. The case where $L 1$ and $L 2$ are both edge lines or inner lines are straightforward.

From this discussion, we see that $L 1$ and $L 2$ cannot be carrying disparate amount of data. Since this is true for any $L 1$ and $L 2$, and we have shown that all nodes in a single line must have equal number of transmissions, we conclude that all nodes must transmit equal number of times. In other words, we necessarily have a per-node fair schedule.

Knowing that an optimal periodic transmission schedule with multihop relaying is necessarily per-node fair, we derive the upper bound on network throughput.

We begin by considering two particularly straightforward cases. The first is the simple case where we have a 2-node network as described in Fig. 8. It is quite obvious that the best we can do is to allow node 1 to always 


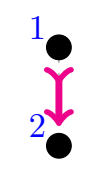

Fig. 8. A 2-node network.

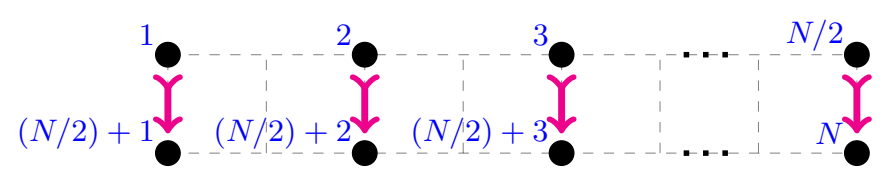

Fig. 9. A regular multiline grid $N$-node network with $N / 2$ lines.

transmit and to constrain node 2 to always receive, according to the transmission schedule $\mathbf{S}^{(1)}$ :

$$
\mathbf{S}^{(1)}=\left[\begin{array}{c}
2 \\
-1
\end{array}\right] \text {. }
$$

The corresponding network throughput is $Y=1$. Next, we consider the case of a regular multiline grid $N$-node network with $N / 2$ lines (see Fig. 9). Here again, it is obvious that the maximum achievable network throughput is provided by the transmission schedule where all the first $N / 2$ nodes always transmit while all the remaining nodes always receive. We therefore see that if $\eta=N / 2$, the optimal network throughput is $Y=N / 2$.

We finally consider the more general case of a regular multiline grid $N$-node network where each line accommodates at least 3 nodes.

Theorem 2. In a regular multiline grid $N$-node network with multihop relaying where $N \geq 3 \eta$ and $\eta \geq 1$, the network throughput is upper bounded by $(N-\eta) / 2$.

Proof: Each line forwards independently its own messages towards the final destination node. Let $\mathbf{S}^{(T)}$ be an optimal transmission schedule. According to theorem $1, \mathbf{S}^{(T)}$ is per-node fair. Thus, every node $i$, where $1 \leq i \leq$ $N-\eta$ transmits at least once during $T$ time slots. Therefore, among the $N T$ entries of $\mathbf{S}^{(T)}$, there exist at least $N-\eta$ positive entries and $N-\eta$ negative entries $\forall T$. The $\eta$ respective final destination nodes of relayed traffic on each line are $N-\eta+1, N-\eta+2, \cdots, N$. Each of these nodes receives at least $(N / \eta)-1$ messages over one period of $T$ time slots, since every line contains $(N / \eta)-1$ transmitting nodes.

Consider a final destination node $h$, where $N-\eta+1 \leq h \leq N$. Node $h-2 \eta$ has transmitted at least one message to its neighbor $h-\eta$ at time slot $t$. Due to the half-duplex constraint, node $h$ remains idle during:

- time slot $t+1$, if node $h-\eta$ is not transmitting at time slot $t$ (the situation is illustrated using one solid red arrow in Fig. 10);

- time slot $t+2$, if node $h-\eta$ transmits also at time slot $t$, since node $h$ receives during time slot $t+2$ the interference coming from node $h-2 \eta$ (the situation is illustrated using two dotted blue arrows in Fig. 10). 


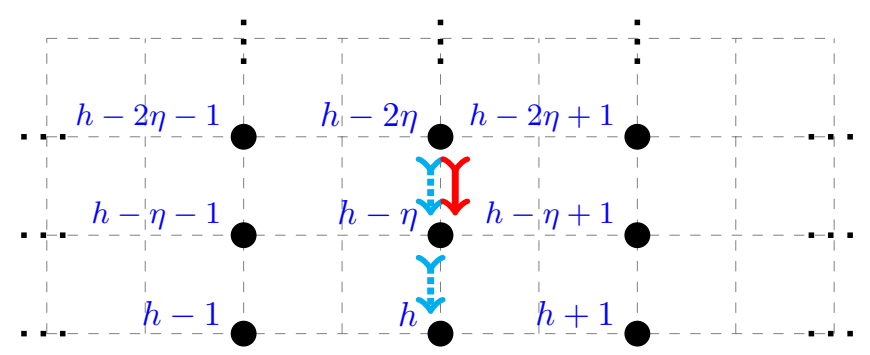

Fig. 10. The activity around a destination node at a given time slot.

Hence, there are at least $\eta$ idle entries in $\mathbf{S}^{(T)} \forall T$.

Now, let us assume $T=1$, i.e., $\mathbf{S}^{(T)}$ is a column vector $(N \times 1)$, and we know the minimum total number of positive, negative and idle entries in $\mathbf{S}^{(T)}$. Consequently, we have $(N-\eta)+(N-\eta)+\eta \leq N$, i.e., $N \leq \eta$. This leads to a contradiction since $N \geq 3 \eta>\eta$. It follows that $T \geq 2$.

Let us consider a source node $k$ such that $1 \leq k \leq \eta$. Node $k$ transmits at least one message to its neighbor $k+\eta$. In turn, node $k+\eta$ transmits at least one message to $k+2 \eta$ at time slot $t^{\prime}$. During $t^{\prime}-1$, node $k$ cannot transmit to avoid interference at $k+2 \eta$, i.e., $k$ remains idle. As a result, there are at least $\eta$ additional idle entries in $\mathbf{S}^{(T)} \forall T$. Furthermore, we look at maximizing the network throughput. Thus far, we know that among the $N \times T$ entries of $\mathbf{S}^{(T)}$, there are necessarily $N-\eta$ positive entries, $N-\eta$ negative entries, and $2 \eta$ idle entries, and we have the period is such that $T \geq 2$. As long as every $\mathbf{S}^{(T)}$ contains at least $2 N=N-\eta+N-\eta+2 \eta$ entries, the problem of maximizing the network throughput can be reduced to minimizing the period $T$, whose minimum value is 2. Therefore, considering the number of receptions accommodated in $\mathbf{S}^{(T)}$, we deduce that $Y \leq(N-\eta) / 2$.

\section{Optimal Transmission Schedules}

After deriving the upper bound on network throughput, we investigate schedules that achieve this limit. 


\section{A. Illustrative transmission schedules}

Consider the schedule $\mathbf{S}^{(4)}$ for a regular 3-line grid 12-node network with multihop relaying, i.e., $N=12$ and $\eta=3$ (see Fig. 11).

$$
\mathbf{S}^{(4)}=\left[\begin{array}{cccc}
4 & 4 & 0 & 0 \\
0 & 0 & 5 & 5 \\
6 & 6 & 0 & 0 \\
7 & -1 & -1 & 7 \\
-2 & 8 & 8 & -2 \\
9 & -3 & -3 & 9 \\
-4 & -4 & 10 & 10 \\
11 & 11 & -5 & -5 \\
-6 & -6 & 12 & 12 \\
-7 & 0 & 0 & -7 \\
0 & -8 & -8 & 0 \\
-9 & 0 & 0 & -9
\end{array}\right]
$$

Counting the total number of receptions (or transmissions) in $\mathbf{S}^{(4)}$, we see that $\mathbf{S}^{(4)}$ achieves the upper bound of $(12-3) / 2=4.5$. $\mathbf{S}^{(4)}$ is a representative schedule that we can use to better understand how to decide on suitable transmissions and how to manage the effects of resulting interferences.

Fig. 11 shows the activity in the network during time slot 2 . The simple gray arrow with dotted line is used to represent interference caused by a transmission during the previous time slot $(t=1)$, while the orange dotted-line arrow with a head and a tail is used to represent interference generated during the current time slot $(t=2)$. Running transmissions are depicted using the solid blue arrows with a head and a tail.

By exploiting propagation delays to favor, on one hand, as many concurrent transmissions as possible, and on the other hand, concentrate interference at unintended nodes, one can maximize network throughput. For instance, in $\mathbf{S}^{(4)}$, the interferences from the neighbors 4,6 and 8 arrive at node 5 during time slot 2 , when it is transmitting.

Fig. 12 describes the activity in the network during time slot 4 . Five simultaneous transmissions during the same time slot is the maximum one can allow in the regular 3-line grid 12-node network.

When formulating a schedule over four time slots, each of the first nodes on odd lines transmits successively two messages and remains idle during the other two time slots. Each of the first nodes on even lines remains idle during two time slots and transmits in turn two messages consecutively. The other nodes receive consequently from their respective downstream neighbors during two time slots, and transmit two messages to their respective upstream neighbors during two time slots, until they reach the final destination nodes. Accordingly, over a period $T=4$, the resulting schedule contains $2(N-\eta)$ transmissions, since except for the $\eta$ final destination nodes, all nodes in the network transmit twice. Hence, the network throughput is $Y=(N-\eta) / 2$. Such a design can be obtained using an appropriate problem formulation and solution described in section $\mathrm{V}$. 


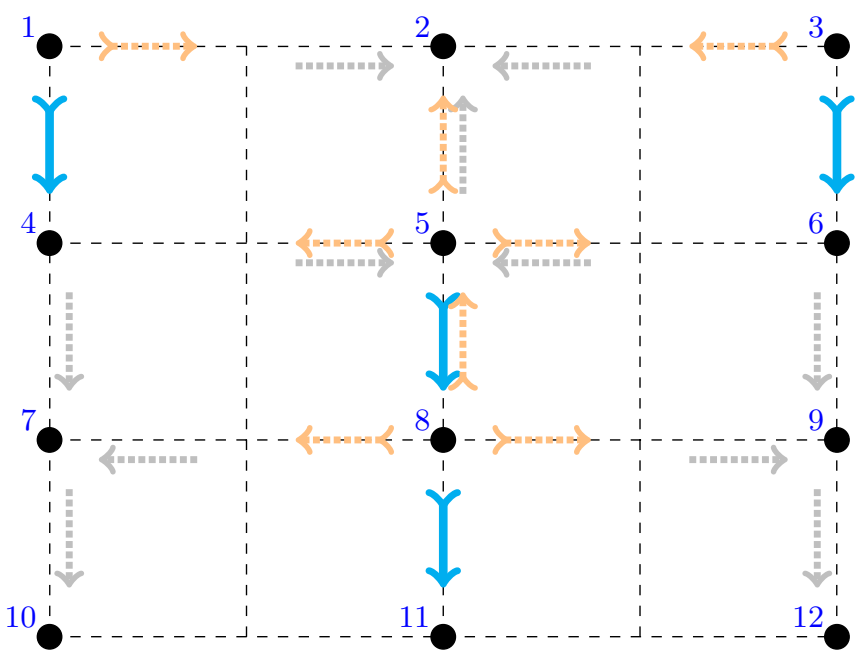

Fig. 11. Useful packets and interferences during time slot 2 in a regular 3-line grid 12-node network, according to $\mathbf{S}^{(4)}$.

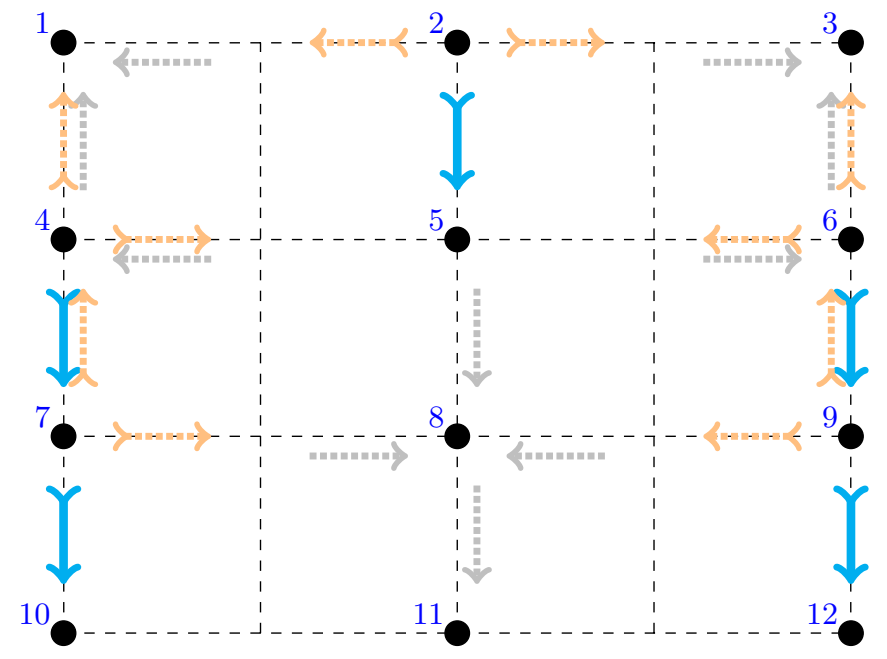

Fig. 12. Useful packets and interferences during time slot 4 in a regular 3-line grid 12-node network, according to $\mathbf{S}^{(4)}$.

\section{B. Grid topology features}

A grid topology is immensely useful for wide area coverage as well as long distance data transmission. Some remote sensing and monitoring applications may consider collecting information only every 2-unit distance instead of every 1-unit distance. Cost savings considerations, especially in large area deployments, are also very critical. Thus, a grid topology with uneven spacing, horizontally and vertically, as has been considered in this paper, is well adapted to applications where the requirements are in collecting information every 2-unit distance. Compared to a regular grid network with 1-unit spacing, horizontally and vertically, this architecture makes it possible to achieve considerable gains. On the one hand, we avoid over-dimensioning the network and deploying unnecessary nodes. 
On the other hand, such a geometry allows us to achieve higher network throughput, since in grid topology with 1-unit spacing, horizontally and vertically, resulting interferences have greater range and greater penalty.

Furthermore, there is another important aspect of this geometry. In view of the multiline structure of the network, we can see it as a collection of multiple lines where the elementary component is a node line, which has, when considered separately, a certain optimal network throughput. The network is built from this collection by considering the existence of reciprocal interference effect between every set of neighboring lines. However, the 2-unit spacing between adjacent lines is a distance that makes it possible to prevent network throughput degradation while pursuing optimal performance on each line. In a regular $N$-node linear architecture with the same network model described above, the maximum achievable network throughput is:

$$
Y=\frac{N-1}{2}
$$

Bringing lines close to each other within the same network induces mutual interferences. Normally, one would expect these interferences to negatively impact the network throughput. However, for the topology studied here, performance is maintained, i.e., in a multiline grid network with $(N / \eta)$ nodes on each line, the maximum achievable network throughput is:

$$
Y=\eta \frac{(N / \eta)-1}{2}=\frac{N-\eta}{2}
$$

which is the optimal network throughput we have demonstrated in the previous section. It is the same network throughput as in an $N$-node network with separate lines, far apart from each other!

\section{Scheduling Algorithm}

\section{A. Problem formulation}

The geometry of an $N$-node network is fully described by the delay matrix $\mathbf{D}$. The size of the network is given by

$$
A=\max _{i, j} \mathbf{D}_{i j}=D_{1 N}
$$

In addition to the delay matrix $\mathbf{D}$, the interference range $2 g$ is a fundamental element in the problem formulation and the solution.

Following [17], we formulate the problem of finding a $T$-periodic optimal schedule, denoted by $\mathbf{S}^{*}$, as a sequential decision problem with finite memory. The current state of the system is known and represented by $\mathbf{S}^{\{t\}}$, which is the partial schedule, given all transmissions occurring between time slots $t-2 g$ and $t-1$. Bear in mind that a transmission will not remain in the network more than $2 g$ time slots. $\mathbf{S}^{\{t\}}$ includes all transmissions made at time slot $t-1$ and all $2 g-1$ time slots earlier. However, resulting receptions and interferences at later time slots, i.e., after $t-1$, are taken into account in the current state $\mathbf{S}^{\{t\}}$. Let $\mathcal{S}$ denote the discrete state space of $\mathbf{S}^{\{t\}}$. Node $i$ is said to be available at time slot $t$, if $i$ is not an intended recipient during $t$. The decision $x_{i}^{\{t\}}$ to be taken at each available node $i$ implies granting access to $i$, i.e., $x_{i}^{\{t\}}>0$, or maintaining $i$ idle, i.e., $x_{i}^{\{t\}}=0$. Let $\mathcal{X}\left(\mathbf{S}^{\{t\}}\right)$ 
denote the set of all possible decisions (also referred to as actions), whereas $\mathcal{X}$ designates the decision space. The effect of action $\mathbf{x}^{\{t\}}$ is represented by the state transition equation (15), which provides the new state $\mathbf{S}^{\{t+1\}}$ :

$$
\mathbf{S}^{\{t+1\}}=\Delta\left(\mathbf{S}^{\{t\}}, \mathbf{x}^{\{t\}}\right) .
$$

The current state $\mathbf{S}^{\{t\}}$ is updated to the next state $\mathbf{S}^{\{t+1\}}$ with the transition function $\Delta($.$) , using transmissions$ in $\mathbf{x}^{\{t\}}$. Recall that the number of transmissions in $\mathbf{x}^{\{t\}}$ is exactly the same as the number of correctly completed transmissions. The transition reward $H$ is nothing other than the number of transmissions introduced by action $\mathbf{x}^{\{t\}}$. It can be written as:

$$
H\left(\mathbf{x}^{\{t\}}\right)=\sum_{i=1}^{N} \mathbb{1}\left(x_{i}^{\{t\}}>0\right), \quad \forall \mathbf{x}^{\{t\}} \in \mathcal{X}\left(\mathbf{S}^{\{t\}}\right) .
$$

By taking optimal actions $\left(\mathbf{x}^{*\left\{t^{\prime}\right\}}, \mathbf{x}^{*\left\{t^{\prime}+1\right\}}, \ldots, \mathbf{x}^{*\left\{t^{\prime}+T-1\right\}}\right)$, we derive an optimal transmission schedule $\mathbf{S}^{*}$. This optimal strategy $X^{*}$ where

$$
\mathbf{x}^{*\left\{t^{\prime}\right\}}=X^{*}\left(\mathbf{S}^{\left\{t^{\prime}\right\}}\right)
$$

allows us to obtain the maximum network throughput (designated by $Y^{*}$ ). Accordingly, using the transition rewards over one period $T$, the value of $Y^{*}$ is obtained:

$$
Y^{*}=\frac{1}{T} \sum_{t^{\prime}=1}^{T} H\left(\mathbf{x}^{*\left\{t^{\prime}\right\}}\right)
$$

Note that there may be more than one optimal strategy.

In terms of the dynamic programming approach used in [17], $Y(\mathbf{S}, \mathbf{x}):(\mathcal{S}, \mathcal{X}) \longrightarrow \mathbb{R}_{+}$can be identified as the objective function having $Y^{*}$ as the best possible value. Moreover, the action value function denoted by $Q(\mathbf{S}, \mathbf{x})$ : $(\mathcal{S}, \mathcal{X}) \longrightarrow \mathbb{R}_{+}$, can be adopted to describe the optimal strategy:

$$
X^{*}(\mathbf{S})=\underset{\mathbf{x} \in \mathcal{X}(\mathbf{S})}{\arg } \max \left(H(\mathbf{x})+Q\left(\Delta(\mathbf{S}, \mathbf{x}), \mathbf{x}^{\prime}\right)\right),
$$

where $\mathbf{x}^{\prime}$ is the decision immediately following $\mathbf{x}$. Note that satisfying the Bellman equation, the action value function can be expressed in its recursive form as

$$
Q(\mathbf{S}, \mathbf{x})=\max _{\mathbf{x} \in \mathcal{X}(\mathbf{S})}\left(H(\mathbf{x})+Q\left(\Delta(\mathbf{S}, \mathbf{x}), \mathbf{x}^{\prime}\right)\right) .
$$

As long as we do not know the true action value function, it can be estimated iteratively at each time slot. To this end, standard algorithms require performing exhaustive state space and decision space enumerations. Thus, the complexity grows very fast with the size of the network. The approximation of the action value function is a more practical alternative. Although it is a sub-optimal technique, it surprisingly achieves high performance. This method is based on the concept of approximate dynamic programming [22].

\section{B. Practical algorithm}

The cardinality of the decision space $\mathcal{X}$ is $\mathcal{O}\left(N^{N}\right)$. We can reduce the decision space by using successive sequential transmission decisions within one time slot [17]. Each decision is represented by a 2-tuple $(k, l)$ for a single transmission from node $k$ to node $l$ at time slot $t$. In this way, the computational complexity of the decision 
space enumeration problem is minimized to $\mathcal{O}\left(N^{3}\right)$. Consequently, we introduce a new numbering scale, designated by $a$, within each time slot $t$. Let $H^{\{t\}}$ denote the number of transmissions in time slot $t$. We have:

$$
a=\left\{\begin{array}{rll}
1,2, \ldots, H^{\{t\}}, & \text { if } & H^{\{t\}}>0 \\
0, & \text { if } & H^{\{t\}}=0 .
\end{array}\right.
$$

After $a-1$ transmission decisions and using the transition function $\Delta($.$) , the partial schedule \overline{\mathbf{S}}^{\{t, a\}} \in \overline{\mathcal{S}}$ is combined with the transmission decision $\overline{\mathbf{x}}^{\{t, a\}}$ to find the next partial schedule, as indicated by

$$
\begin{aligned}
& \overline{\mathbf{S}}^{\{t, a+1\}}=\Delta\left(\overline{\mathbf{S}}^{\{t, a\}}, \overline{\mathbf{x}}^{\{t, a\}}\right), \quad \forall a<H^{\{t\}} \\
& \overline{\mathbf{S}}^{\{t+1,1\}}=\Delta\left(\overline{\mathbf{S}}^{\left\{t, H^{\{t\}}\right\}}, \overline{\mathbf{x}}^{\left\{t, H^{\{t\}}\right\}}\right) .
\end{aligned}
$$

In agreement with the new formulation of the problem within the state space $\overline{\mathcal{S}}$ and the decision space $\overline{\mathcal{X}}$, we introduce an action value function:

$$
\begin{aligned}
\bar{Q}(\overline{\mathbf{S}}, \overline{\mathbf{x}}) & =\max _{\overline{\mathbf{x}} \in \overline{\mathcal{X}}(\overline{\mathbf{S}})}\left(H(\overline{\mathbf{x}})+\bar{Q}\left(\Delta(\overline{\mathbf{S}}, \overline{\mathbf{x}}), \overline{\mathbf{x}}^{\prime}\right)\right), \\
& =\max _{\overline{\mathbf{x}} \in \overline{\mathcal{X}}(\overline{\mathbf{S}})} \bar{Q}\left(\Delta(\overline{\mathbf{S}}, \overline{\mathbf{x}}), \overline{\mathbf{x}}^{\prime}\right)+1,
\end{aligned}
$$

since $H(\overline{\mathbf{x}})=1$ is the reward for the single transmission decided within the considered time slot. Note that at the stage shown by (24), only transmission decision $\overline{\mathbf{x}}$ matters in the optimal action finding process, unlike $\overline{\mathbf{x}}^{\prime}$.

As stated above, the true value of $\bar{Q}$ may be obtained using computationally expensive techniques such as relative value iteration. Nevertheless, their complexity makes them unfeasible, especially for networks with a significant size $A$. As a practical and much simpler alternative, an appropriate approximation of the value function can be developed on the basis of our comprehension of the problem structure and properties.

We have seen that the time extent of the current state is $2 g$. Similarly, any upcoming transmission will act on the network within $2 g$ time slots, and we seek to maximize the number of future transmissions. Therefore, one must go through a state that allows this. Given a transmission decision $\overline{\mathbf{x}}$, the capacity of a state to accommodate future transmissions within $2 g$ time slots is used thus far as an approximate measure of the state-action pair value.

The whole $N$-node network consists of partially-overlapping collision domains. Let us enumerate first the constraints on transmission in UWA environment with such conditions. Given the partial schedule $\overline{\mathbf{S}}^{\{t\}}$ at time slot $t$, a single transmission from node $k$ to node $l$ at time slot $t^{\prime} \geq t$ is allowed only if:

- node $l$ is within the transmission range of node $k$, i.e., $D_{k l} \leq g$;

- it is not a self-transmission, i.e., $l \neq k$;

- there is no transmission or reception already planned for node $k$ at $t^{\prime}$, i.e., $\bar{S}_{k, t^{\prime}}^{\{t\}}=0$;

- there is no transmission or reception already planned for node $l$ at $t^{\prime}+D_{k l}$, i.e., $\bar{S}_{l, t^{\prime}+D_{k l}}^{\{t\}}=0$;

- there is no interference at node $l$ at $t^{\prime}+D_{k l}$, originating from any other node $i\left(i \neq k\right.$ and $\left.D_{i l} \leq 2 g\right)$ at $t^{\prime}+D_{k l}-D_{i l}$, i.e., $\nexists i$ s.t. $\left[D_{i l} \leq 2 g\right] \&\left[\bar{S}_{i, t^{\prime}+D_{k l}-D_{i l}}^{\{t\}}>0\right]$

- the transmission from node $k$ will not cause interference at any other node $j\left(j \neq l\right.$ and $\left.D_{k j} \leq 2 g\right)$ at $t^{\prime}+D_{k j}$ while $j$ is receiving a message from node $i\left(D_{i j} \leq g\right), \quad$ i.e., $\quad \nexists i, j \quad$ s.t. $\quad\left[D_{k j} \leq 2 g\right] \&\left[D_{i j} \leq\right.$ 
$g] \&\left[\bar{S}_{i, t^{\prime}+D_{k j}-D_{i j}}^{\{t\}}=j\right]$.

We use the transmission indicator function $C_{k l \delta}$ to determine if a transmission from node $k$ to node $l$ at $t^{\prime} \geq t$ is allowed, given the partial schedule $\overline{\mathbf{S}}^{\{t\}}$ at $t$. The action value function approximation should take into consideration $C_{k l \delta}\left(\overline{\mathbf{S}}^{\{t\}}\right)$, which can be expressed as:

$$
C_{k l \delta}\left(\overline{\mathbf{S}}^{\{t\}}\right)=\left\{\begin{array}{lll}
0, & \text { if } \quad D_{k l}>g \\
0, & \text { if } \quad l=k \\
0, & \text { if } \quad \bar{S}_{k, t^{\prime}}^{\{t\}} \neq 0 \\
0, & \text { if } \quad \bar{S}_{l, t^{\prime}+D_{k l}}^{\{t\}} \neq 0 \\
0, & \text { if } \quad \exists i \text { s.t. }\left[D_{i l} \leq 2 g\right] \& \\
& & {\left[\bar{S}_{i, t^{\prime}+D_{k l}-D_{i l}>0}^{\{t\}}>0\right.} \\
0, & \text { if } \quad \exists i, j \text { s.t. }\left[D_{k j} \leq 2 g\right] \& \\
& {\left[D_{i j} \leq g\right] \&\left[\bar{S}_{i, t^{\prime}+D_{k j}-D_{i j}}^{\{t\}}=j\right]} \\
1, & \text { otherwise, }
\end{array}\right.
$$

where $\delta=t^{\prime}-t$.

Thus, the action value function approximation $\bar{Q}$ is given by:

$$
\bar{Q}\left(\Delta\left(\overline{\mathbf{S}}^{\{t\}}, \overline{\mathbf{x}}\right), \overline{\mathbf{x}}^{\prime}\right)=\sum_{k=1}^{N} \sum_{l=1}^{N} \sum_{\delta=0}^{2 g} C_{k l \delta}\left(\Delta\left(\overline{\mathbf{S}}^{\{t\}}, \overline{\mathbf{x}}\right)\right),
$$

where $\overline{\mathbf{x}}^{\prime}$ is the decision immediately following $\overline{\mathbf{x}}$. Recall that only decision $\overline{\mathbf{x}}$ matters in the expression of $\bar{Q}$.

Furthermore, to observe the multihop relaying process, another constraint is used. We update the decision space $\overline{\mathcal{X}}$ to $\ddot{\mathcal{X}}$ before making use of the action value function in order to orient all transmissions in one direction, i.e., from nodes $1,2, \cdots, \eta$ toward final destination nodes $N-\eta+1, N-\eta+2, \cdots, N$, respectively. This constraint can be described as:

$$
\ddot{\mathcal{X}}=\{(i, j) \in \overline{\mathcal{X}} \quad \text { s.t. } \quad i<j\} .
$$

On the other hand, looking at the optimal network throughput $(N-1) / 2$ in the case of a regular linear architecture (i.e., with no interference impact from adjacent lines), we are intuitively led to consider an additional constraint. We should ensure that the number of transmission decisions per time slot does not exceed the average of $(N-\eta) / 2$ transmissions. Otherwise, a large number of transmissions during a certain time slot would penalize the decisions at the following time slots, due to resulting receptions and mainly resulting interferences. This constraint can be expressed using the following condition:

$$
H^{\{t\}} \leq\left\lceil\frac{N-\eta}{2}\right\rceil
$$

where $\lceil b\rceil$ gives the smallest integer greater than or equal to $b$.

For $N \geq 3 \eta$ and $\eta \geq 2$, Algorithm 1 summarizes the above discussed steps to make pertinent transmission decisions at time slot $t$ and updates the transmission schedule. In addition to the delay matrix $\mathbf{D}$ and the current 


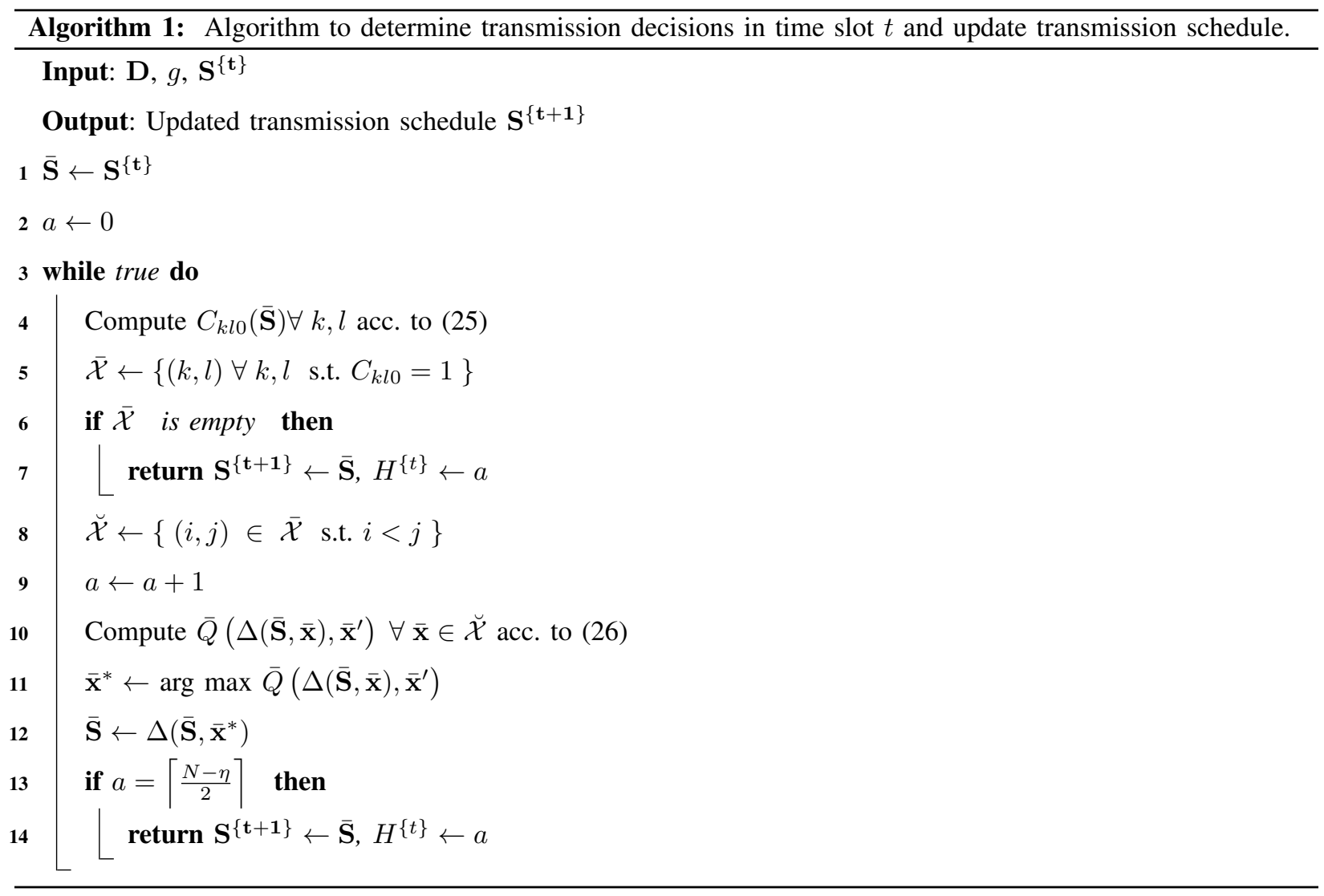

transmission schedule $\mathbf{S}^{\{\mathbf{t}\}}$, the transmission range $g$ is a required input for the algorithm. The values of the two parameters $N$ and $\eta$ are also used in the algorithm, but they can be obtained directly from delay matrix $\mathbf{D}$.

Given the partial schedule $\mathbf{S}^{\{t\}}$, the algorithm goes through the following operations and successively repeats them until there are no more allowable transmissions for that time slot. First, it explores all possible transmission decisions in time slot $t$. After that, the decision space found is updated using the multihop relaying constraint. Next, the approximate action value function is invoked to find the optimal transmission decision. It is a transmission that has only a small effect on forthcoming transmissions. As soon as the number of transmissions reaches $\lceil(N-\eta) / 2\rceil$, the algorithm updates the partial transmission schedule and moves to the next time slot $t+1$.

This algorithm can be seen as an extension of the algorithm proposed in [17] that is adapted to regular multiline grid networks with multihop relaying. It needs a very short time to run and yields optimal schedules for networks of any size. The algorithm has been successfully tested on regular multiline grid networks with several values for $N$ and $\eta$. We summarize results from several runs of the algorithm for various $N$ and $\eta$ in Fig. 13. The throughput consistently achieves the upper bound $(N-\eta) / 2$. For illustration purposes, note that in the case where $N=25$ and $\eta=5$, the optimal solution is found in less than two seconds using a basic laptop.

For larger $N$ (up to 60 ) and $\eta$ (up to 10), different configurations can be made and all the algorithm simulation runs result in the optimal solution, i.e., the throughput consistently achieves the upper bound $(N-\eta) / 2$. 


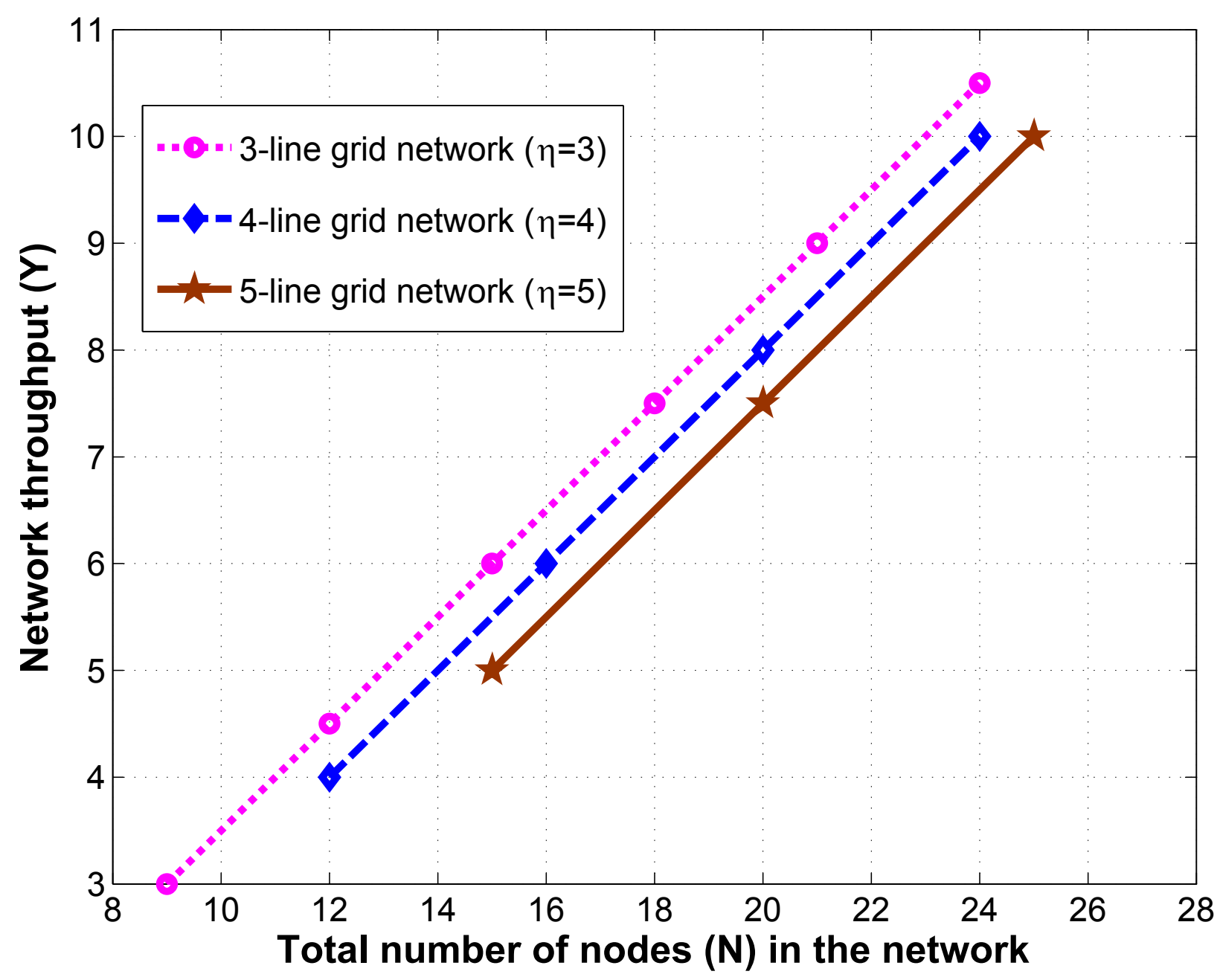

Fig. 13. Network throughput for schedules generated using Algorithm 1 for various $N \leq 25$ and $\eta=3$ to 5 . The throughput consistently achieves the upper bound $(N-\eta) / 2$.

\section{CONCLUSions \& FUture Work}

This paper considered TDMA-based MAC protocols that take advantage of large propagation delays to achieve maximum network throughput in regular multiline grid networks with multihop relaying. We demonstrated that an optimal schedule is necessarily per-node fair, and derived the upper bound on network throughput. Furthermore, we presented a computationally efficient algorithm to find optimal schedules regardless of the number of the lines and the size of the network.

This study provides substantial results with regards to multiline grid topologies with multihop relaying. However, in order to understand, at a fundamental level, how advantageous nonzero delays are in such geometries, it would be worthwhile to explore the linear topology with respect to traffic policy, collision domain size, and various fairness constraints. 


\section{ACKNOWLEDGMENT}

This work has been supported in part by the Fonds unique interministériel (FUI) France within the COMET project.

\section{REFERENCES}

[1] J. Heidemann, M. Stojanovic, and M. Zorzi, "Underwater Sensor Networks: Applications, Advances, and Challenges," Philosophical Transactions of the Royal Society (A), pp. 158-175, Jan. 2012.

[2] W. Zhang, M. Stojanovic, and U. Mitra, "Analysis of a Linear Multihop Underwater Acoustic Network," IEEE Journal of Oceanic Engineering, vol. 35, no. 4, pp. 961-970, Oct. 2010.

[3] Y. Xiao, M. Peng, J. Gibson, G. Xie, D.-Z. Du, and A. Vasilakos, "Tight Performance Bounds of Multihop Fair Access for MAC Protocols in Wireless Sensor Networks and Underwater Sensor Networks," IEEE Transactions on Mobile Computing, vol. 11, no. 10, pp. 1538-1554, Oct. 2012.

[4] S. Ergen and P. Varaiya, "PEDAMACS: Power Efficient and Delay Aware Medium Access Protocol for Sensor Networks," IEEE Transactions on Mobile Computing, vol. 5, no. 7, pp. 920-930, July 2006.

[5] K. Kredo, P. Djukic, and P. Mohapatra, "Cooperative Multihop Communication for Underwater Acoustic Networks," in Proc. 1st ACM Int. Workshop Underwater Networks (WUWNet), Los Angeles, CA USA, Sep. 2006.

[6] A.-K. Othman, W. Wan Zainal Abidin, H. Zen, K. Ping, and N. Zamhari, "Performance Evaluation for Network Set Up and Nodes Discovery Protocol in Underwater Grid Topology Networks," in 8th Asia-Pacific Symposium on Information and Telecommunication Technologies (APSITT), Kuching, Sarawak, Malaysia, June 2010.

[7] A. Reza and J. Harms, "Robust Grid-based Deployment Schemes for Underwater Optical Sensor Networks," in IEEE 34th Conference on Local Computer Networks (LCN), Zurich, Switzerland, Oct. 2009.

[8] X. Che, I. Wells, P. Kear, G. Dickers, X. Gong, and M. Rhodes, "A Static Multi-hop Underwater Wireless Sensor Network Using RF Electromagnetic Communications," in IEEE 29th International Conference on Distributed Computing Systems (ICDCS), Montreal, Quebec, Canada, June 2009.

[9] C.-C. Hsu, K.-F. Lai, C.-F. Chou, and K. Ching-Ju Lin, "ST-MAC: Spatial-Temporal MAC Scheduling for Underwater Sensor Networks," in Proc. IEEE INFOCOM, the 28th IEEE International Conference on Computer Communications, Rio de Janeiro, Brazil, April 2009.

[10] Y. Guan, C.-C. Shen, and J. Yackoski, "MAC Scheduling for High Throughput Underwater Acoustic Networks," in Proc. IEEE Wireless Communications and Networking Conf. (WCNC), Cancun, Quintana-Roo Mexico, Mar. 2011.

[11] L. Badia, M. Mastrogiovanni, and M. Zorzi, “An Optimization Framework for Joint Sensor Deployment, Link Scheduling, and Routing in Underwater Sensor Networks," in Proc. 1st ACM Int. Workshop on Underwater Networks (WUWNet), Los Angeles, CA USA, Sep. 2006.

[12] F. Presti, C. Petrioli, R. Petroccia, and A. Shashaj, "A Scalable Analytical Framework for Deriving Optimum Scheduling and Routing in Underwater Sensor Networks," in 9th IEEE International Conference on Mobile Ad hoc and Sensor Systems (MASS), Las Vegas, NV USA, Oct. 2012.

[13] B. Peleato and M. Stojanovic, "Distance Aware Collision Avoidance Protocol for Ad-hoc Underwater Acoustic Sensor Networks," IEEE Communications Letters, vol. 11, no. 12, pp. 1025-1027, Dec. 2007.

[14] X. Guo, M. Frater, and M. Ryan, "Design of a Propagation-Delay-Tolerant MAC Protocol for Underwater Acoustic Sensor Networks," IEEE Journal of Oceanic Engineering, vol. 34, no. 2, pp. 170-180, April 2009.

[15] K. Kredo, P. Djukic, and P. Mohapatra, "STUMP: Exploiting Position Diversity in the Staggered TDMA Underwater MAC Protocol," in Proc. IEEE INFOCOM 2009, Rio de Janeiro, Brazil, Apr. 2009.

[16] H.-H. Ng, W.-S. Soh, and M. Motani, “A Bidirectional-Concurrent MAC Protocol With Packet Bursting for Underwater Acoustic Networks,” IEEE Journal of Oceanic Engineering, vol. 38, no. 3, pp. 547-565, July 2013.

[17] M. Chitre, M. Motani, and S. Shahabudeen, "Throughput of Networks With Large Propagation Delays," IEEE Journal of Oceanic Engineering, vol. 37, no. 4, pp. 645-658, Oct. 2012.

[18] D. Jing, L. Ben, and P. Varshney, "Tuning the Carrier Sensing Range of IEEE 802.11 MAC," in Proc. IEEE Global Telecommunications Conf., Dallas, Texas USA, Nov. 2004. 
[19] S. Boppana and J. Shea, "Overlapped Carrier-Sense Multiple Access (OCSMA) in Wireless Ad Hoc Networks," IEEE Transactions on Mobile Computing, vol. 8, no. 3, pp. 369-383, Mar. 2009.

[20] Y. Noh, P. Wang, U. Lee, D. Torres, and M. Gerla, "DOTS: A propagation Delay-aware Opportunistic MAC protocol for underwater sensor networks," in Proc. 18th IEEE International Conference on Network Protocols (ICNP), Kyoto, JAPAN, Oct. 2010.

[21] D. Shukla, L. Chandran-Wadia, and S. Iyer, "Mitigating the Exposed Node Problem in IEEE 802.11 Ad hoc Networks," in Proc. 12th IEEE International Conference on Computer Communications and Networks (ICCCN), Dallas, TX USA, Oct. 2003.

[22] W. B. Powell, Approximate Dynamic Programming: Solving the Curses of Dimensionality. New York: Wiley-Interscience, 2007. 